\title{
Seclusion and Restraint Using Among Psychiatric Patients
}

\author{
Qusai Mohammed Harahsheh* \\ School of Nursing, The Hashemite University, Jordan
}

*Corresponding author: Qusai Mohammed Harahsheh, School of Nursing, The Hashemite University, Jordan.

Received Date: June 25, 2019

Published Date: July 09, 2019

\section{Introduction}

Every person in our world adapt with live challenges in different way, but some people cannot develop ways to cope with this challenge which resulted to mental disorders among these persons as a chain of destructive behaviors images. In general, mental illness are inability to cope with different stressors which developed by environment internally or externally, this failure of coping reflected as incongruent feelings, thoughts and behaviors with norms which used locally or culturally, on the same time this maladaptive interfere with people function socially, physically and occupationally [1]. Mental and psychiatric science developed many methods to control this destructives behaviors such as medication, technique, and seclusion, the point here about using seclusion from legal and ethical dimensions, is it acceptable or not? Seclusion defined as involuntary isolation of patient in specific room, this room named as seclusion room, and has many characteristics focusing on non-stimulating place, it must be locked, supervised by window, and contain the safety measurements as a hole (Health Care Commission, 2008), On the other hand the mental health commission defined the seclusion as a place has locked door designed in way which prevent going outside, this person stay in this room alone and on a specific time.

The Evidence Based Practice considered as the best way to develop any action or any decision during caring process, the integration of clinical expertise, patient values, and the best research evidence into the decision-making process for patient care. Clinical expertise refers to the clinician's cumulated experience, education and clinical skills. The patient brings to the encounter his or her own personal preferences and unique concerns, expectations, and values. The best research evidence is usually found in clinically relevant research that has been conducted using sound methodology.
The current author used PICOT question which reflect Population, Intervention, Comparison, Outcome and Time to clarify seclusion and physical restrain effectiveness. According to PICOT question: The first question: among psychiatric patients' seclusion is effective in controlling aggressiveness comparing with physical restrain during treatment period? The second question: among psychiatric patients' seclusion is effective in controlling aggressiveness comparing with not using seclusion during treatment period? The purpose of this study is to clarify the effectiveness of using seclusion and physical restrain to control aggressive behaviors among mentally ill patients in psychiatric sittings.

\section{Literature Review}

This literature review will explain and clarify the effectiveness of using seclusion and physical restrain in controlling aggressiveness among mentally ill patient in psychiatric sittings and describe the power and limitations of every study depending on specific standers and items in specific tables. Actually seclusion and restraint still widely used in our world, an Italian systematic review study examine the effect of coercive procedures to control the aggressive behaviors among psychiatric patients in different psychiatric sittings, this systematic review use 74 studies after excluding 768 study which didn't met the author criteria, the study found that there are a variations of using seclusion and restraint from center to another [2], similar prevalent were founded in united states, Australia, Germany and Switzerland [3]. On the other hand, the review founded that seclusion and restraint used among females more than male patients, and among borderline or antisocial personality disorder higher than other psychiatric disorders [4]. 
Furthermore, in case control study in Denmark which includes 235.000 patients between violent and non-violent psychiatric patients using seclusion and restraint, the authors founded that there is increasing in using seclusion and restraint among female patients who have mania, experiencing drug abuse or alcohol abuse more than other psychiatric patients [5]. About voluntary and involuntary admission to psychiatric sittings [6], they founded that there is a positive correlation between restraint using and involuntary admission cases, and to be more specific they found that $11 \%$ of 282 patients faced seclusion and restraint; $9.5 \%$ of them faced seclusion and $1.8 \%$ of them faced restrain, concluded by retrospective cohort study. Other study founded that 424 patients have been secluded from 1214 in voluntary admission cases which reflected $35 \%$ of admission cases as a hole, and 117 cases was restrained which also reflected $10 \%$ from same sample [7]. Related to aggression, a prospective study on 148 aggressive patients, $70 \%$ of them faced restrain which mean that there is a strong positive correlation between aggressions and restrain [8]. Another systematic review in UK on 2007, focused on interventions used to decrease using of seclusion and restrain, this systematic review worked on randomized control trails of non-pharmacological interventions. Moreover, an old study shown that seclusion and restrain highly effective to control aggressive behaviors and protect patients and staff [9], on the other hand.

Other studies in 2005 founded that seclusion and restrain have harmful effects and traumatic experiences to the patients and staff. The systematic review focused on regulations and policies changes in UK and concluded that there are tighter controls on how, when and where seclusion and restrain should be used, on the same time this standard should followed by post-seclusion debriefings with staff and patients [10].

On the other hand focusing on support level such as regular visits to the patients in psychiatric sittings, frequent active listening to the patients, discussion between staff and patients, individual crises preventions plans, peer to peer support; all of them help to reduce seclusion and restrain especially when we taking about child and adolescent in-patients [11]. Moreover, many ideas may help to stop or at least reduce using of seclusion and restraint focused on leadership seclusions such as sitting new expectations to staff [12], reassess policies [13], change practice system on way which put seclusion and restraint as a last choice in treatment process [14]. On the same point [10] founded that increasing number of staff playing major role in reducing use of seclusion and restraint, other study find that using of cross-displenarry staff such as nurses and social workers can positively affect caring process by decrease using of seclusion and restraint by providing support for those patients [15].

Furthermore staff education considered as a central point of caring process and of decreasing seclusion and restrain cases $[10,12,15]$, on this point education divided to two main dimensions the first one focusing on new care model which working on decrease conflict incidences and high-therapy [16], the second dimension focused on alternative behavioral intervention off education which working on problem solving and de-escalating techniques [17].

Moreover, to decrease needs of seclusion, the psychiatric emergency response team was founded, this team present in many states' hospitals [10], this team skillful and have extra training related to rapid effective actions, qualified in using verbal deescalating technique and conflict resolving skills [18]. Another new systematic review and quantitative synthesis in Australia 2013, worked on reducing seclusion and restraint among disable people, they founded that health care providers can reduce restrain among disable people who have aggression, agitation and self-harm attempts, on the same time the literatures concluded that when health care providers know the reason of self-harm and aggression then they can develop ideas to prevent or at least reduce seclusion and restraint using. On the other hand the literature founded that there is a differences between period of using restrain between day and night shift duty in psychiatric sittings, to be more specific they founded that $81 \%$ reduction of median time of restrain in AM shift and $10 \%$ reduction of median time of restrain in PM shift [19].

Furthermore, there is a redactor from $54 \%$ to $11 \%$ of using mechanical restrain by inerratic of behavior in terveations programs from 59 to 124 program as organization behavior of manger it, on the other hand there is no changing on injuries percentages which resulted for aggression behavior [20].

To be more focus on changes among am and pm shift [21] use three phases in they trail to measure the presenting of decreafiy seclusion and restrain usage among psychiatric patients, the phase classified as active phase, training phase, practice phase, depending on single subject, multiple - baseline design and 23 staff members, 20 individuals who have intellectual disabilities, the staff worked on 12cession training program about verbal redirection, , verbal exchanges which lead to aggression and stat medications, finally they founded that there is a reduction on incident of seclusion and restraint using from 2.67/week in AM shift to 2.00/week, and 2.67/ week to 1.50 /week in PM shift after training phase, and to 0.20 / week in AM shift and 0.35/week in PM shift after practice phase.

Another systematic review working to assess the effectiveness and the level of safety of seclusion and restrain among adult psychiatric in-patients as a short term violence management in psychiatric sittings and emergency departments, the review done in united kingdom in 2006 and include systematic reviews, qualitative studies and before-after studies, the other collect all researches which related to the main topic from 1985 to 2002, and concluded that there are insufficient evidences available to detect if restrain and seclusion considered as safe short time intervention for aggressive and violated patients in the psychiatric and emergency sittings. On the other hand, the systematic review founded that the seclusion and restrain must use as the last choice of intervention after failing of the service users to response to other alternatives and other claiming situations measures demonstrated to the patients without any effective results. 
Regarding to [22] they advocate to reduce using of seclusion and restrain as a type of treatments for mentally ill patients, on the same time and according to Royal Collage of Psychiatry in UK; there is no sufficient evidences to consider restrain and seclusion safe and effective intervention must use or not among psychiatric and mentally ill patients, on the other hand a small old, descriptive study suggest that using of seclusion and restrain help to develop safe caring process and decrease of violence incident [23]. On the same point [24] suggest in their descriptive study that using management participation, education and improving staff patterns play very important role in reduce using seclusion and restrain among psychiatric patients. About the relationship between death and restrain, three experimental studies founded that there is no relationship between prone position restrain and death; which mean that restrain is safe from this dimension. [25-27].

Another review worked on restrain and seclusion among serious mentally ill patients published by John Wiley and Sors in 2012 in Finland, the literature worked on comparing between the effectiveness of seclusion and restrain with other alternative measures, on the same time the literature worked on examine effects of strategies which used to prevent using of seclusion and restrain. The review founded that there are many unwanted harm effects, other studies shown that there is improvement in control for aggression by using medication [28]. On the other hand the literature founded that no controlled studies support using of seclusion and restrain among psychiatric and mentally ill patients, moreover the review founded that using of seclusion and restrain may lead to increasing of mortality and morbidity more than other alternative choices such as drug and non-drug approaches. Moreover a systematic review of restraint intervention for challenging behaviors among persons who have intellectual disabilities which applied in UK 2015, the author used research syntheses which divided to meta-analysis and qualitative meta synthesis techniques, the review amid to examine if restrain interventions are effective in reducing challenging behaviors for intellectual disable patients, finally and after using 76 related studies they concluded that restrain interventions behaviors can be improved by specific model while Appling it for intellectual disable patients.

\section{Discussion}

After collecting a huge number of articles, the current author founded that many articles founded that seclusion is effective to control aggressiveness more than physical restrain during treatment period among psychiatric patients, strongest design found the same results but its advocate to apply other alternatives. On the same time, old studies founded that seclusion is effective comparing non seclusion, but updated studies founded that there are many harm effects of using seclusion, the current author with the second idea because the sample size where higher and the date of articles was more updated.

\section{Recommendations}

Seclusion ethical dilemma which discussed in this paper guide the current author to develop important recommendation should be followed in the near future especially in Jordan, the first one focused on staff education about communication skills and deescalating technique to eliminate all factors which push the staff to use seclusion. The second one focused on pharmacological education for staff to use a a-typical antipsychotic medication rather than seclusion on the same time provide courses about signs of agitation and aggressiveness of patients to take the correct precautions and interventions to prevent increasing symptoms severity which considered the only rational to use seclusion. Finally, the new science focused on providing training and courses for staff who working on psychiatric field to enhance their ability to use other measurement with psychiatric patient especially with aggressive and violated patients to decrease seclusion usage in psychiatric sittings.

\section{Summary and Conclusion}

The main reason to use seclusion is to protect patient and others safety as most of studies mentioned, on the other hand there are many other alternatives may use to meet this goal. To be more specific there is no specific and clear law to prevent using seclusion in psychiatric sittings as the current author searching process, but there are huge studies advocate to decrease using seclusion related to ethical considerations. Finally, the new science direction is focusing on providing trainings and courses about communication skills de-escalating techniques and other alternative measure for staff who are working on psychiatric field to decrease using of seclusion.

\section{Acknowledgment}

The current author would like to gratefully acknowledge his direct instructor Mr. Asem Alhmoud MSN, RN, all other instructors at Hashemite University, especially the mental health department in the faculty of nursing, all health care providers' team at the Jordanian Center for Psychiatric Health.

\section{Conflict of Interest}

No conflict of interest.

\section{References}

1. Townsend MC (2006) Essentials of psychiatric mental health nursing: Concepts of care in evidence-based practice Philadelphia, PA: FA Davis, (ed.).

2. Betemps EJ, Somoza E, Buncher CR (1993) Hospital characteristics, diagnoses, and staff reasons associated with use of seclusion and restraint. Hosp Community Psychiatry 44: 367-371.

3. Irving K (2004) Inappropriate restraint practices in Australian teaching hospitals. Aust J Adv Nurs 21: 23-27.

4. Beck NC, Durrett C, Stinson J, Coleman J, Stuve P, et al. (2008) Trajectories of seclusion and restraint use at a state psychiatric hospital. Psychiatr Serv 59: 1027-1032.

5. Benjaminsen S, Gotzsche Larsen K, Norrie B, Harder L, Luxhoi A (1996) Patient violence in a psychiatric hospital in Denmark. Rate of violence and relation to diagnosis. Nord J Psychiatry 50: 233-242.

6. Bilanakis N, Kalampokis G, Christou K, Peritogiannis V (2010) Use of. coercive physical measures in a psychiatric ward of a general hospital in Greece. Int J Soc Psychiatry 56: 402-411.

7. Husum TL, Bjørngaard JH, Finset A, Ruud T (2010) A cross-sectional 24. prospective study of seclusion, restraint and involuntary medication in 
acute psychiatric wards: patient, staff and ward characteristics. BMC Health Serv Res 10 p: 89

8. Klimitz H, Uhlemann H, Fähndrich E (1998) Are restraints used too. frequently? Indications, incidence and conditions for restraint in a general psychiatric department. A prospective study. Psychiatr Prax 25: 235-239.

9. Cotton NS (1995) Seclusion as therapeutic management: an invited commentary. American Journal of Orthopsychiatry 65: 245-248.

10. Smith GM, Davis RH, Bixler EO (2005) Pennsylvania State Hospital system's seclusion and restraint reduction program. Psychiatr Serv 56: 1115-1122.

11. LeBel J, Stromberg N, Duckworth K (2004) Child and adolescent inpatient restraint reduction: a state initiative to promote strength-based care. J Am Acad Child Adolesc Psychiatry 4343: 37-45.

12. Sullivan AM, Bezmen J, Barron CT (2005) Reducing restraints: alternatives to restraints on an inpatient psychiatric service - utilizing safe and effective methods to evaluate and treat the violent patient. Psychiatr Q 76: 51-65.

13. Fisher WA (2003) Elements of successful restraint and seclusion reduction programs and their application in large, urban, statepsychiatrichospital in a large, urban, state psychiatric hospital. J Psychiatr Pract 99: 7-15.

14. Schreiner GM, Crafton CG, Sevin JA (2004) Decreasing the use of mechanical restraints and locked seclusion. Administration and Policy in Mental Health 3131: 449-463.

15. Donovan A, Siegel L, Zera G (2003) Child \& adolescent psychiatry: Seclusion and restraint reform: an initiative by a child and adolescent psychiatric hospital. Psychiatr Serv 54: 958-959.

16. Bowers L, Brennan G, Flood C (2006) Preliminary outcomes of a trial to reduce conflict and containment on acute psychiatric wards: City Nurses. J Psychiatr Ment Health Nurs 13: 165-172.

17. Taxis JC (2002) Ethics and praxis: alternative strategies to physical restraint and seclusion in a psychiatric setting. Issues Ment Health Nurs 23: $157-170$.
18. D Orio BM, Purselle D, Stevens D (2004) Reduction of episodes of seclusion and restraint in a psychiatric emergency service. Psychiatr Serv 55: 581-583.

19. Jensen CC, Lydersen T, Johnson PR, Weiss SR, Marconi MR, et al. (2012) Choosing staff members reduces time in mechanical restraint due to self-injurious behaviour and requesting restraint. J Appl Res Intellect Disabil 25: 282-287.

20. Williams DE, Grossett DL (2011) Reduction of restraint of people with intellectual disabilities: An organizational behavior management (OBM) approach. Research in Developmental Disabilities 32: 2336-2339.

21. Singh NN, Lancioni GE, Winton AS W, Singh AN, Adkins, AD, et al. (2009) Mindful staff can reduce the use of physical restraints when providing care to individuals with intellectual disabilities. Journal of Applied Research in Intellectual Disabilities 22: 194-202.

22. Salias E, Fenton M (2000) Seclusion and restraint for people with serious mental illnesses. Cochrane Database Syst Rev 2: CD001163.

23. Hafner RJ, Lammersma J, Ferris R, Cameron M (1989) The use of seclusion: A comparison of two psychiatric intensive care units. Aust N Z J Psychiatry 23(2): 235-239.

24. Kingdon DG, Bakewell EW (1988) Aggressive behaviour: Evaluation of a non-seclusion policy of a district psychiatric service. Br J Psychiatry 153: 631-634.

25. Parkes J (2000) Sudden death during restraint: A study to measure the effect of restraint positions on the rate of recovery from exercise. Med Sci Law 40(1): 39-44.

26. Schmidt P, Snowden T (1999) The effects of positional restraint on heart rate and oxygen saturation. J Emerg Med 17: 77-82.

27. Chan TC, Vilke GM, Neuman T, Clausen JL (1997) Restraint position and positional asphyxia. Ann Emerg Med 30: 578-586.

28. Janssen WA, Noorthoorn EO, De Vries WJ, Hutschemeakers GJM, Lendemeijer HHGM et al. (2008) The use of seclusion in the Netherlands compared to countries in and outside Europe. International Journal of Law and Psychiatry 31(6): 463-470. 